\title{
Mental Health and Social Contact During the COVID-I 9 Pandemic: An Ecological Momentary Assessment Study
}

This is a preprint that has not been peer-reviewed. Last updated: January II 202 I.

\author{
Eiko I. Fried' ${ }^{\oplus}$, and Faidra Papanikolaou', \& Sacha Epskamp ${ }^{2,3}$
}

\begin{abstract}
The COVID-19 pandemic caused once-in-a-lifetime disruptions of daily life for many students, such as closure of university. In March 2020, during the beginning of the outbreak in the Netherlands, we used Ecological Momentary Assessment to follow 80 bachelor students 4 times per day for 14 days, assessing mental health, social contact, and COVID-19 related variables. Despite rapidly increasing rates of infections and deaths, anxiety, loneliness, and COVID-19 related concerns decreased, especially in the first few days. Other mental health variables, such as stress levels, remained stable, while depressive symptoms increased. Despite social distancing measures implemented by the Dutch government halfway through our study, students showed no changes in the frequency of in-person social activities. Dynamic network models identified vicious cycles between mental health variables and being alone, which predicted concerns about COVID-19 and was followed by further mental health problems. Findings and implications are discussed in detail.
\end{abstract}

\section{Keywords}

anxiety; COVID-19; depression; ecological momentary assessment; mental health; network model; resilience; stress; time series

\footnotetext{
'Leiden University, Faculty of Social and Behavioral Sciences, Leiden, The Netherlands 2University of Amsterdam, Department of Psychology, Amsterdam, The Netherlands

${ }^{3}$ Centre for Urban Mental Health, Amsterdam, The Netherlands
}

Corresponding Author:

Eiko I. Fried, Leiden University, Faculty of Social and Behavioral Sciences, Leiden, The Netherlands.

Email: eikofried@gmail.com. Twitter: @eikofried. Online: www.eiko-fried.com, www.psych-networks.com. 


\section{Introduction}

About $75 \%$ of all severe mental health problems develop before the age of 24 (Kessler et al., 2005), and many studies have documented that students report consistently higher levels of mental health problems than the general population (Denovan \& Macaskill, 2016; Gaspersz et al., 2012; Mortier, Auerbach, et al., 2018; Stallman, 2010; Tomoda et al., 2000; Tran et al., 2017; Williams et al., 2018). Paired with the fact that student samples are convenient to recruit, this has led to a considerable body of research on understanding, predicting, and preventing stress and mental disorders in students.

The WHO Mental Health Survey documented 1-year incidence rates of mental illness among students of about 20\%, with anxiety and mood disorders among the most prevalent problems (Auerbach et al., 2016). The College Health Intervention Project estimated that one in four college students experience symptoms of depression, and one in ten suicidal thoughts (Mackenzie et al., 2011). In data of over 2,500 college freshmen collected in Belgium, the 1-year incidence rates were about 7\% for Major Depression (Ebert et al., 2018), 5\% for suicidal thoughts and behaviors (Mortier, Demyttenaere, et al., 2018), and 10\% for non-suicidal self-injury (Kiekens et al., 2019). Longitudinal work indicates that problems increase during the first semester in freshmen (Besser \& Zeigler-hill, 2014). Mental health problems in students are related to considerable impairment of functioning, decreased academic performance and life satisfaction, higher levels of physical comorbidities, increased college dropout, and increased levels of smoking, alcohol, and drug abuse (Ebert et al., 2018; Ribeiro et al., 2017).

While the literature on student mental health has been growing rapidly, only a minority of work consists of prospective stress studies that follow participants during periods of considerable stress (Bolger et al., 2000), such as residency in medical students (Fried et al., 2014; Guthrie et al., 1998). Of those, there are only a handful of studies to date that used daily diary or Ecological Momentary Assessment (EMA) methods, e.g. via smart phones, to assess the impact of stressors on students' daily lives. The few existing studies have focused primarily on US presidential elections and other US events like the Las Vegas shooting (Frank et al., 2019; Roche \& Jacobson, 2019).

The etiology of mental health problems in students is diverse, and includes stressors such as including financial problems (Heckman et al., 2014), academic pressure (Misra \& McKean, 2000), adjusting to new social and geographical environments (Montgomery \& Cote, 2003), relationships, life-stage transitions, and time management (Wilks et al., 2009). One massive recent stressor is the COVID-19 pandemic, and early work indicates increases of mental health problems across the globe (Ettman et al., 2020; Jacobson et al., 2020; Nelson et al., 2020; Twenge \& Joiner, 2020). In the Netherlands, where we collected our data, the pandemic led to severe disruptions of public life. Requests for public transport information in March dropped by 75\%, while the number of COVID19 infections and deaths grew rapidly from 10 to 12,595 and 0 to 1,038, respectively (Figure 1). The pandemic was accompanied by public health measures announced by the Dutch government (The Dutch Government, 2020), potentially causing novel, once-in-a-lifetime stressors for students, including: ban of public gatherings; ban of non-essential international travel; closing of, among others, universities, schools, restaurants, cinemas, and gyms; shortage of some basic supplies due to mass purchases; health concerns about family and friends; and economic concerns. Our goal was to study the impact of these stressors on student mental health.

We followed 80 bachelor students enrolled at Leiden University closely over the course of 2 weeks, using EMA. After a battery of questions at baseline, we queried students about mental health problems, social contact and isolation, and concerns about the pandemic. We concluded the study with a short exit survey, including information on COVID-19 diagnosis, mental health, implementation of social distancing and personal hygiene behaviors, and whether students felt well informed by Leiden University and the Dutch government.

This data allows us to answer three questions. First, what is the general frequency of mental health problems, social behaviors, and pandemic-related concerns in the 2 weeks following a university shut down-and do these variables change over time? Second, what variables predict changes in mental health over the 2-week study period? And third, what are the potential causal relations among these variables (e.g., do concerns COVID-19 lead to higher levels of mental health problems at the next measurement point)? We answer the last question by estimating dynamic 
network models, consistent with the network approach to psychopathology (Borsboom, 2017; Fried \& Cramer, 2017; Robinaugh et al., 2019).

Given the novelty of the COVID-19 pandemic and little previous work studying prospective stressors via EMA, we have no a priori hypotheses except that the pandemic has adverse effects on student mental health. Our primary goal for this paper is to faithfully report on the collected data in an exploratory way. We share all data, code, and measures in the supplementary materials (https://osf.io/mvdpe/).

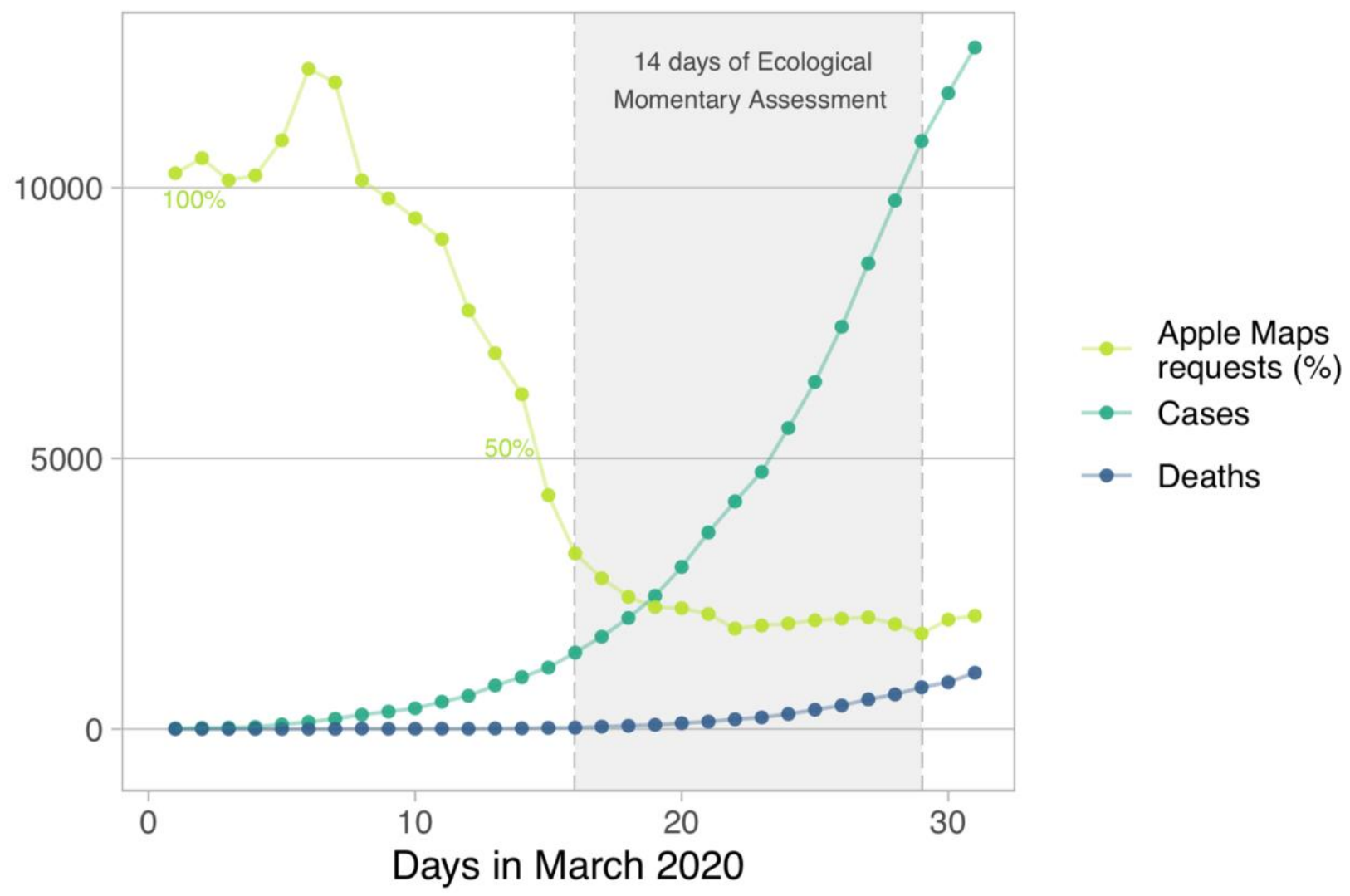

Figure I: We conducted a 2-week prospective stress study in the Netherlands. We plot Apple Maps requests for public transport in the Netherlands as proxy for disruption of normal daily life, in \% compared to January 2020 (from: https://www.apple.com/covid 19/mobility), as well as increase of COVID- 19 cases and deaths in the Netherlands (https://www.worldometers.info/coronavirus/country/netherlands).

\section{Procedure}

\section{Methods}

The study took place between March 11 and April 04 2020, and was conducted in three parts. First, participants completed a 45-minute baseline assessment, required to continue to the second stage: 2 weeks of EMA. This stage lasted from Monday March 16 (University closed on Friday March 13) to Sunday March 29. During this period, participants received a prompt on their smart phones four times per day (noon, $3 \mathrm{pm}, 6 \mathrm{pm}$, and $9 \mathrm{pm}$ ). Each assessment lasted approximately 2.5 minutes, and participants had to answer the prompt within 60 minutes, after which it expired. Third, participants completed a 20-minute post-assessment survey, available from March 29 to April 5. No 
manipulations took place. All assessments were conducted via Ethica Data, a data collection platform available on both Android an iOS that participants installed on their smart phones.

\section{Participants}

We recruited participants in the week following March 4, through online advertisements on social networks, posters and flyers distributed in the Faculty of Social Sciences of Leiden University, or directly approached by the researchers. Participants were students at Leiden University, and were reimbursed for participation with study credits and the chance to win one of four $25 €$ vouchers. Students received credits proportional to completed EMA surveys. Out of the 100 initially recruited participants, 84 completed the baseline survey, 79 completed the EMA surveys and 80 completed the post assessment; we include the 80 participants who completed both pre and post assessments in this study. The study was approved by the Ethics Board of Leiden University, Faculty of Social and Behavioral Sciences.

Of the 80 students, 60 identified as female, 19 as male, and 1 as other, with an age mean of 20.38 (SD=3.68, range 18-48), and a mix of 19 nationalities, the most common of which were Dutch $(N=36)$, German $(N=16)$, and Finnish $(N=7)$. Most students were single $(N=50)$, fewer in a relationship $(N=27)$ or married $(N=3)$, and the large majority of students were first-year bachelor students $(N=68)$ enrolled in psychology $(N=70)$. Seventeen students reported having suffered from mental health problems in the past, or having taken psychiatric drugs; 26 students were employed. To assess the representativeness of our sample compared to Leiden University bachelor students in general, we were able to obtain information on sex, age, and nationality for all 2,039 psychology bachelor students enrolled at Leiden University 2019-2020. Age mean was 21.32 (range 17-54), with 1,573 female $(77 \%)$ and 466 male (23\%) students; this compares well to our sample $(75 \%$ female, $24 \%$ male, $1 \%$ other). Of these students 1,394 were Dutch (68\%), which means that our sample (48\% Dutch) oversampled international students. This is likely because our recruitment and study was carried out in English language.

\section{Measures}

All measures can be found in the supplementary materials. Importantly, the current paper only reports on a number of selected items of specific interest for our research questions; full data is available online, with the exception of some data we deleted to guarantee anonymity.

\section{Baseline and follow-up assessments}

The baseline survey consisted of 159 questions, in which participants were asked, among others, about their age, gender, relationship status, employment status, nationality, and prior mental health issues. In addition, we assessed several constructs for which we used in part adapted or shortened versions of original scales to decrease participant burden: last-week problems regarding depression, anxiety, and stress (Depression Anxiety Stress Scale; DASS-21; (Lovibond \& Lovibond, 1995)); last-month perceived stress (10-item Perceived Stress Scale (Cohen \& Williamson, 1988); general loneliness (5 items from the 8-item revised UCLA loneliness scale; ULS-8 (Russell et al., 1980)); general frequency of social in-person activities (1 item); and self-efficacy (10-item General Self-Efficacy Scale (Schwarzer, R., \& Jerusalem, 1995); All scales were modified to range from 1 through 5 for consistent assessment. Due to these differences compared to the original scales, we refer to the adapted DASS-21 score as Global Mental Health score, and the adapted ULS loneliness scale as loneliness score, for the remainder of the manuscript.

During follow-up, we queried participants on 74 items. These comprised COVID-19 related symptoms, diagnoses of the participant, diagnosis of close family members and friends; perceived impact on mental health, and whether actions taken by Leiden University had impact on their stress levels; and how well-informed students felt by Leiden University and the Dutch government. In addition, we queried students again on our adapted DASS-21 and loneliness scales.

\section{$E M A$}

For all 14 variables, we queried participants how much, over the last 3 hours, they endorsed a certain feeling or behavior (not at all, slightly, moderately, very, extremely), or how much time they 
spend on a certain activity ( 0 minutes, 1-15 minutes, 15-60 minutes, 1-2 hours, over 2 hours); see Table 1. The mental health items were adapted from the DASS-21/DASS-42 (Lovibond \& Lovibond, 1995)and the Generalized Anxiety Disorder Scale (Spitzer et al., 2006); other items were created for the purpose of this study, based on our experiences with prior EMA studies we conducted in 2018 and 2019 with student populations in the Netherlands.

Table I. Ecological Momentary Assessment items, queried 4 times per day over 2 weeks.

\begin{tabular}{cll}
\hline$\#$ & Abbreviation & Item \\
\hline I & Relax & I found it difficult to relax \\
2 & Irritable & I felt (very) irritable \\
3 & Worry & I was worried about different things \\
4 & Nervous & I felt nervous, anxious or on edge \\
5 & Future & I felt that I had nothing to look forward \\
6 & Anhedonia & I couldn't seem to experience any positive feeling at all \\
7 & Tired & I felt tired \\
8 & Alone & I felt like I lack companionship, or that I am not close to people \\
9 & Social_offline & I spent__on meaningful, offline, social interaction \\
I0 & Social_online & I spent_ using social media to kill/pass the time \\
II & Outdoors & I spent_outside (outdoors) \\
I2 & Cl9_occupied & I spent_occupied with the coronavirus (e.g. watching news \\
thinking about it talking to friends about it) \\
I3 & CI9_worry & $\begin{array}{l}\text { I spent_ thinking about my own health or that of my close } \\
\text { friends and family members regarding the coronavirus }\end{array}$ \\
I4 & Home & I spent_ at home (including the home of parents/partner)
\end{tabular}

Note: Range of all items I-5. Items I through 8: not at all, slightly, moderately, very, extremely. Items 9 through 14: Omin, I-I5 minutes, 15-60 minutes, I-2 hours, over 2 hours.

\section{Statistical analyses}

All analyses were carried out in the free statistical environment R. Data and syntax are available in the supplementary materials.

We used paired t-tests, along with Cohen's D as an effect size measure, to investigate whether DASS-21, the three DASS-21 subscales (depression, anxiety, and stress), and loneliness changed in the 2 weeks between baseline and exit surveys. We estimated a multiple regression model to predict changes in DASS-21 from pre to post, using the predictors gender, age, nationality, relationship status, working, prior mental health issues, self-efficacy, perceived stress, loneliness, and being socially active, controlling for DASS-21 at baseline. Missing data for the above analyses were deleted listwise.

For estimation of dynamic network models, we used two-step multilevel vector autoregression (two-step mIVAR; (Epskamp et al., 2018)), a model in which all variables at a given timepoints are regressed on variables of the previous assessment. Predictors are within-person centered and sample means are added as predictors to separate within- and between-person variance; residuals are used in a second step to investigate contemporaneous relationships. This leads to two networks. (1) The temporal network, that estimates lag-1 associations between all items 
after controlling for all other lagged associations. This provides statistical relations that can be interpreted as Granger-causal: how well does an item predict other items at the next time point after taking into account all other variables (Granger, 1969). (2) The contemporaneous network, that partials out all temporal relations, and then estimates the unique relations among all items within the same time window. A third network returned by two-step mIVAR, the between-persons network, was not investigated due to our relatively low sample size. We visualized the results of network models in graphs that contain nodes (variables) and edges (statistical relationships described above). The temporal model features directed edges, the contemporaneous network undirected edges. Stronger relations are depicted as thicker and more saturated edges; positive edges are blue, negative red. Two-step mIVAR requires stationarity. We detrended the data by fitting fixed-effects linear regression models to each variable, regressing out a linear trend on day number (i.e. general increases in variables over time), and a categorical effect on measurement per day (i.e. fluctuations of variables from morning to evening), at an alpha of 0.05 .

The obtained linear trends from the detrending procedure (i.e. the question whether slopes were different from 0) were utilized in determining whether EMA items changed over time. To investigate whether individuals differed in these trends, we used univariate multilevel regression models, including fixed and random effects, detailed in the supplementary materials.

\section{Sample descriptives}

\section{Results}

Of the 80 students, 60 identified as female, 19 as male, and 1 as other, with an age mean of 20.38 (SD=3.68, range 18-48), and a mix of 19 nationalities, the most common of which were Dutch $(N=36)$, German $(N=16)$, and Finnish $(N=7)$. Most students were single $(N=50)$, fewer in a relationship $(N=27)$ or married $(N=3)$, and the large majority of students were first-year bachelor students $(N=68)$ enrolled in psychology $(N=70)$. Seventeen students reported having suffered from mental health problems in the past, or having taken psychiatric drugs; 26 students were employed. Abbreviations for all EMA items discussed in the subsequent sections are detailed in Table $\mathbf{1}$.

\section{Mental health comparison of baseline and exit surveys}

We identified no changes for DASS-21 scores in the 2 weeks from baseline $(M=15.03$, $\mathrm{SD}=9.08)$ to study exit $(\mathrm{M}=14.57, \mathrm{SD}=8.75), t(76)=0.45, p=0.66, d=0.05$. Analyzing the three DASS21 subscales revealed significant increases for depression from $M=4.46$ ( $S D=3.80$ ) to $M=5.62$ ( $\mathrm{SD}=4.29), t(76)=2.33, p=0.02, d=0.27$; significant decreases for anxiety from $\mathrm{M}=3.64(\mathrm{SD}=3.22)$ to $\mathrm{M}=2.73(\mathrm{SD}=2.85), t(76)=2.87, p=0.005, d=0.33$; and no significant changes for stress from $\mathrm{M}=6.93$ $(\mathrm{SD}=4.09$ ) to $\mathrm{M}=6.22(\mathrm{SD}=3.74), t(76)=1.72, p=0.09, d=0.20$. According to the DASS-21 cutoffs, depression scores increased from the "normal" to the "mild depression" range; anxiety scores decreased from the "mild anxiety" to the "normal" range; and stress scores stayed in the "normal" range. Interestingly, we saw decreases for loneliness scores from pre $(M=11.65, S D=3.92)$ to post $(\mathrm{M}=10.86, \mathrm{SD}=3.90), t(76)=3.14, p=0.002, d=0.36$.

We predicted change in DASS-21 scores from pre to post by gender $(N=60$ female, $N=19$ male), age, nationality ( $N=36$ Dutch, $N=44$ international), relationship status ( $N=50$ single, $N=30$ partnered), working ( $N=54$ no, $N=26$ yes), prior mental health issues ( $N=59$ no, $N=17$ yes), selfefficacy $(M=28.9, S D=3.93)$ perceived stress $(M=30.34, S D=3.36)$, loneliness $(M=11.65, S D=3.93)$, and in-person social activities $(M=3.78, S D=1.3)$. None of the variables predicted significant changes, except for a negative coefficient for DASS-21 at baseline that we controlled for $(b=-0.66, \mathrm{t}(62)=-5.12$, $p<0.001$; overall, $\mathrm{F}(11,62)=3.71, p<0.000$, adjusted $\mathrm{R}^{2}=0.29$ ). The negative relation can likely be explained by regression to the mean, where higher baseline scores predict decreases, i.e. negative changes.

\section{EMA variables}

Investigating For the 14 EMA variables over 56 time points, 6,026 of the 61,208 data points (9.9\%) were missing. Abbreviations for EMA items, and characteristics of change over time, are detailed in Table 1 and Figure 2. Overall, 10 variables significantly decreased over the 2 weeks, one 
variable significantly increased (Home), and three variables (Anhedonia, Social_offline, Outdoors,) remained stationary. Note that these are analyses at the group level; a detailed analysis on how items changed over time on the idiographic can be found in the supplementary materials. We observed the largest decreases for the items C19_occupied (standardized coefficient -0.18), C19_worry (-0.16), Nervous (-0.13), and Worry (-0.12), all $p<0.001$; the increase for Home was $0.03(p=0.03)$. It may appear contradictory if only considering $p$-values that Home increased when Outdoors remained stable, but the standardized coefficients were the same $(-0.03, \mathrm{p}=0.08)$. We also identified significant cyclic patterns within days for the five variables Tired, Social_Offline, Outdoors, Home $(p<0.001)$, and Worry $(p=0.02)$.

We plotted in detail how each item evolved over time (see supplementary figures). Zooming into the item with the strongest decrease, C19-occupied, reveals that on day 1, nearly no students indicated the lowest response category, while nearly half of the students endorsed this response on the last day of the study 2 weeks later (Figure 3). We used univariate multi-level regression models to obtain information on inter-individual differences, and found that $83 \%$ of the students showed a decrease for C19-occupied, 57.6\% a decrease with a standardized coefficient below -0.1. Results were similar for C19-worry, with $76.2 \%$ and $50.6 \%$, respectively. This means that decreases on the group-level were not driven by few individuals. These analyses also provided evidence for large heterogeneity across participants in auto-regressive effects, described in more detail in the supplementary materials. For 10 of the 14 EMA variables, models with random effects on the lagged variables were preferred over simple models with fixed effects.

\section{Network model}

The evolution of means over time in Figures 2 and 3 indicated some strong patterns, for instance, there seemed a strong inverse relation between the means of Social_offline and Home. To investigate these relations further, we estimate contemporaneous and temporal network models that depict conditional dependence relations of all variables, see Figure 4.

In the contemporaneous network (i.e. relations among items within the last 3-hour duration of a given beep), we identified many expected relations among items, such as a positive relation between C19_occupied and C19_worry; and negative relations between Outdoors and Home, Social_offline and Social_online, and Alone and Social_offline. Further, we found that mental health items (with the exception of Tired) were interrelated; Alone was related to (concerns about) Future as well as Anhedonia; and Outdoors was positively (and Home negatively) related to Social-offline, indicating meaningful in-person activities took place at home more than outside.

In the temporal network (i.e. lag-1 relations from one 3-hour measurement period to the next), we identified positive autoregressive coefficients for all nodes ranging from 0.13 (Outdoors) to 0.22 (Alone); positive relations among mental health variables; and some vicious cycles, e.g. between (worry about) Future and Anhedonia, (worry about) Future and Alone, (unable to) Relax and Anhedonia, as well as (unable to) Relax and Social-Online. The most interesting feedback loop was between: Alone $\rightarrow$ C19-worry $\rightarrow$ C19-occupied $\rightarrow$ Anhedonia $\rightleftarrows$ (worry about) Future $\rightleftarrows$ Alone; C19-occupied as part of this cycle also predicted a range of other mental health problems. Both (unable to) Relax and (worry about) Future led to less time spent outside at the next timepoint, and (worry about) Future also negatively predicted Social-offline. Nervous was followed by participants being less Alone, and, interestingly, by lower levels of Anhedonia at the next measurement point.

\section{Post assessment}

At the post assessment, $19.5 \%$ of students indicated that they had had symptoms during the last 3 weeks that could indicate a COVID-19 infection, such as fever, cough, or shortness of breath; none had received a formal diagnosis, however. Only 4 students indicated that a close friend or relative had received a COVID-19 diagnosis. Participants further indicated, on a 5-point Likert scale ranging from 1 (totally disagree) to 5 (totally agree), with 3 being neutral, that: they started washing their hands more frequently during the study period $(M=3.49$ [3.32-3.67]); they avoided social activities with many people $(M=3.70$ [3.56-3.84]); the pandemic impacted their mental health negatively ( $M=3.34$ [3.06-3.62]); they felt somewhat well informed by Leiden University ( $M=3.39$ 
[3.11-3.67]) and the Dutch government ( $M=3.39$ [3.11-3.67]); and that the actions taken by Leiden University and the government had had no impact on their stress levels ( $M=2.88$ [2.60-3.16]).
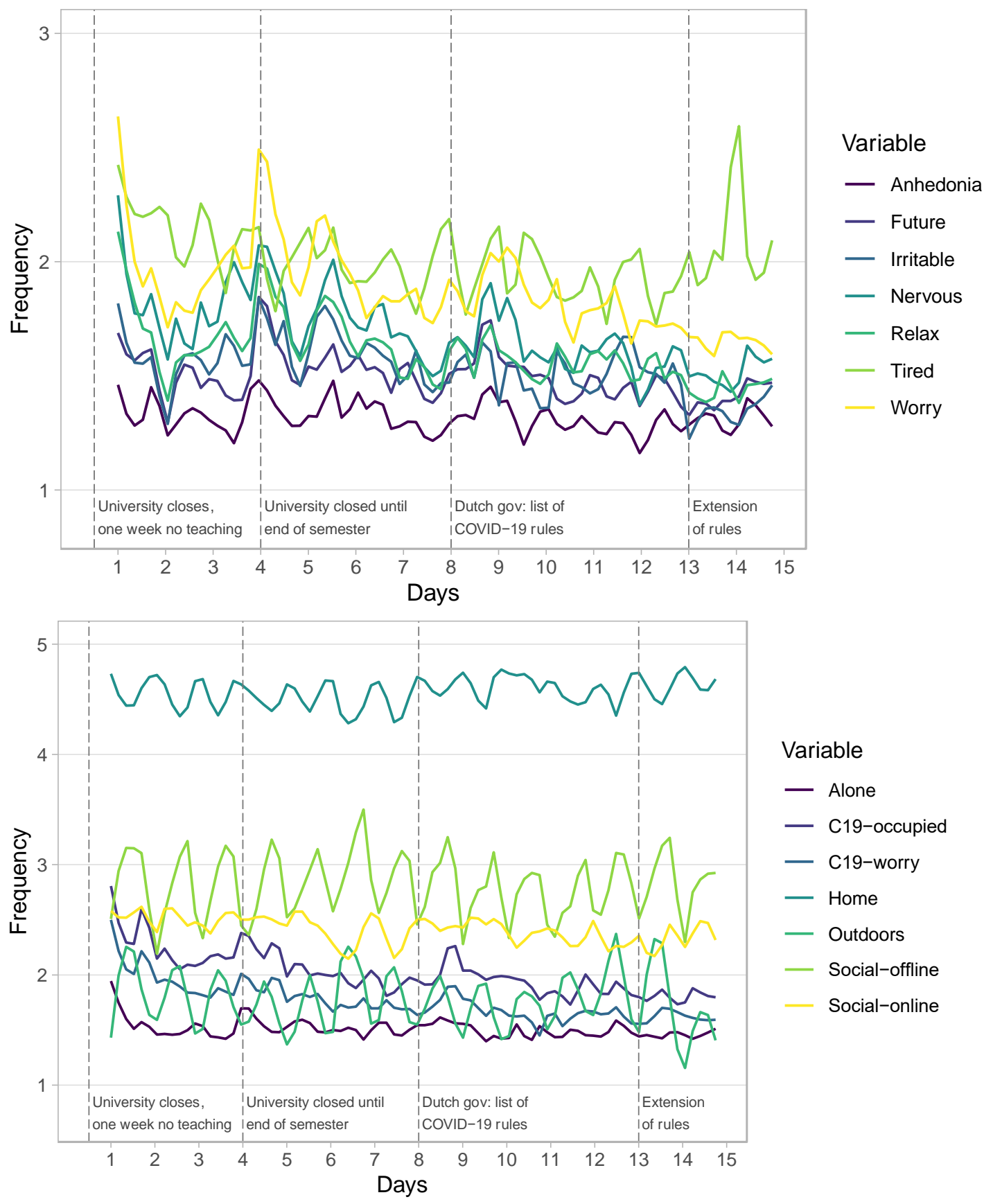

Figure 2. Means of I4 Ecological Momentary Assessment items, assessed 4 times per day over I4 days on a I-5 scale (higher scores: more severe or frequent). Top: mental health related variables. Bottom: social and COVID-I9 related variables. Note that we adapted the $y$-axis range in the upper figure to increase interpretability. Detailed item descriptions can be found in Table I. 

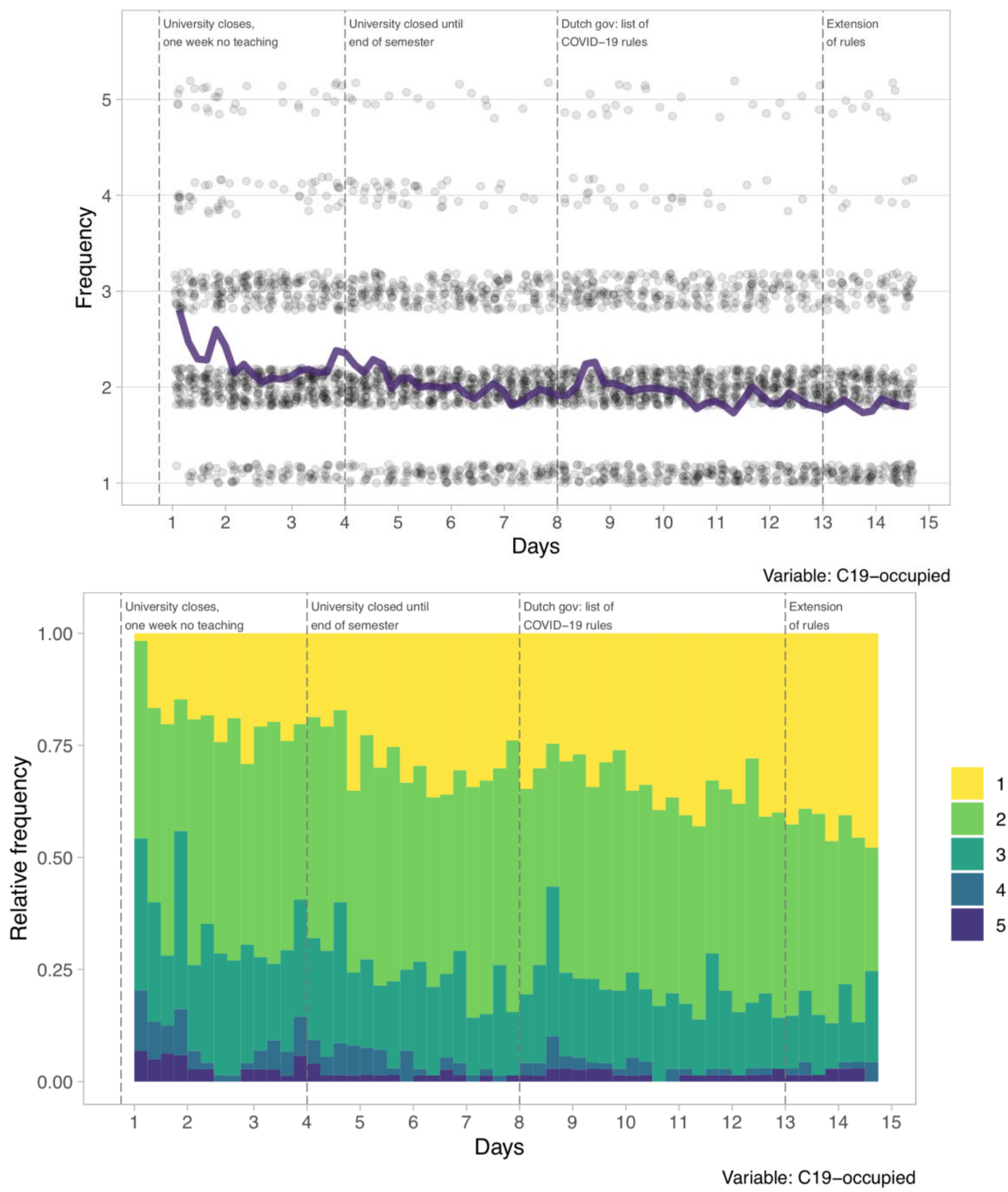

Figure 3. Time course of the Ecological Momentary Assessment item "in the last 3 hours, I spent occupied with the coronavirus (e.g. watching news, thinking about it, talking to friends about it)", which exhibited the largest decreases of all items in our study, with the answer options I $(0 \mathrm{~m}$.), 2 (I I5m.), 3 (I5-60m.), 4 (I-2hrs.), and 5 (>2hrs.). Top: individual item responses per day as dots, plus mean score over time. Bottom: Relative frequencies of ordinal responses. Small peaks can be 
observed on days 4 and 8, potentially following announcements of Leiden University and the Dutch government.
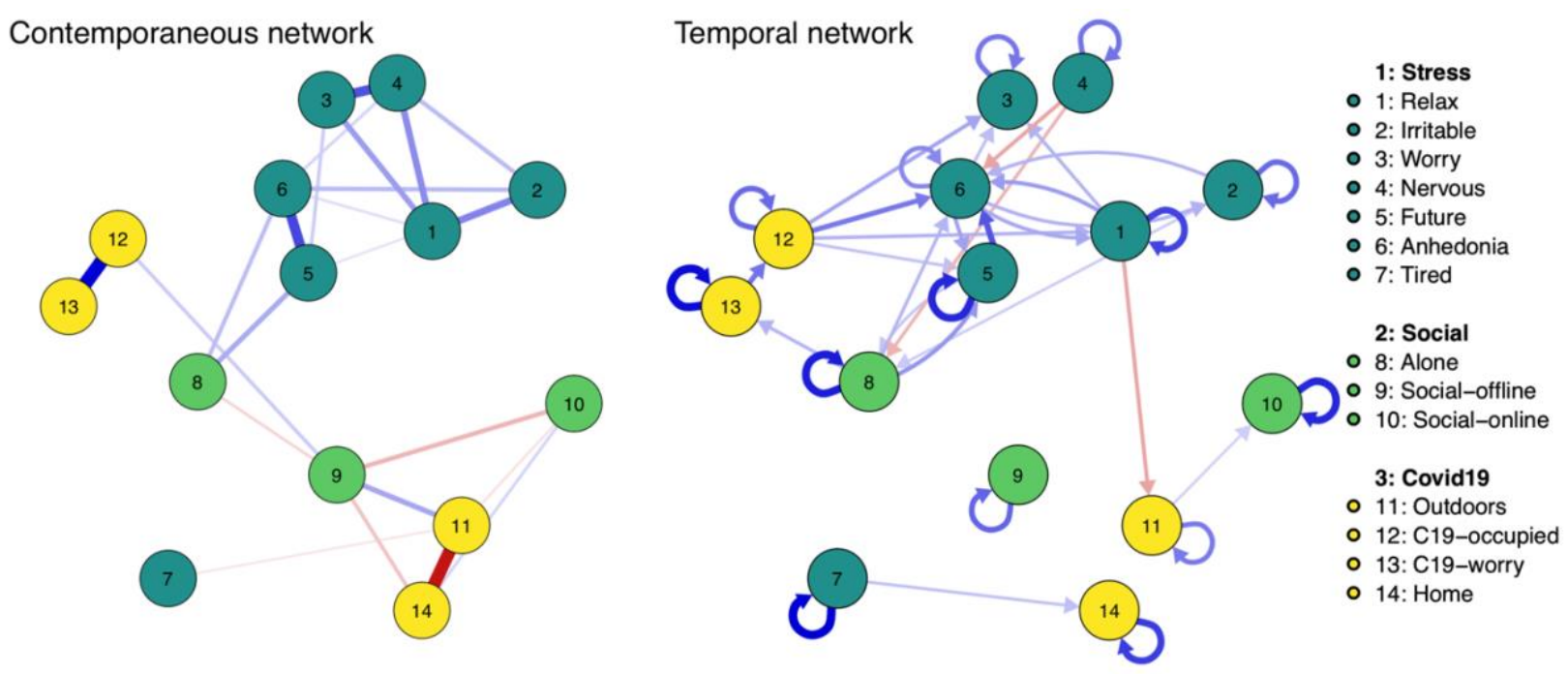

Figure 4. Contemporaneous (left) and temporal (right) relations among I 4 Ecological Momentary Assessment variables gathered 4 times a day over the course of 2 weeks, estimated with a multilevel vector-autoregressive model and depicted as a graph where nodes are variables, and edges are partial correlations among variables. Thicker and more saturated edges depict stronger relations; positive relations in blue, negative relations in red.

\section{Discussion}

We We closely follow 80 students during the early days of the COVID-19 pandemic in the Netherlands, during a time of fairly dramatic changes. Many of these were once-in-a-lifetime disruptions of students' daily lives. The unique nature of the COVID-19 pandemic, as well as our unique EMA data, make the results somewhat difficult to compare to prior work. Nonetheless, in the following section we summarize our main results and connect them to other research.

Generally, we observed somewhat heterogenous changes, with more decreases than increases in mental health problems, both when comparing baseline to exit surveys, and when investigating changes of EMA items during our two weeks of study duration. Only the depression subscale of the DASS-21 increased, from an average in the normal range to an average in the mild depression range. Prior work reported considerable adverse effects on mental health during outbreaks, such as during the Severe Acute Respiratory Syndrome (SARS) epidemic in 2003; among others, being in quarantine, and knowing people with SARS diagnoses, predicted adverse effects (Hawryluck et al., 2004). COVID-19 mental health studies reported increases in depression symptoms (in US adults; Ettman et al., 2020), anxiety symptoms (in young English adults; Kwong et al., 2020), and distress (McGinty et al., 2020) from before to during the pandemic (see also Daly et al., 2020; Twenge \& Joiner, 2020); a study of Dutch children and adolescents reported similar results (Luijten et al., 2020). None of the students in our sample received a COVID-19 diagnosis during our data collection period, and only four participants reported having close friends or family members with diagnoses, which may have mitigated adverse mental health outcomes. Further, scores of our 
participants on the DASS-21 at baseline were overall in the normal or slightly increased range, indicating a sample that may be more resilient to adverse events.

We saw decreases for most mental health related EMA items, including items concerning students' worry about their own health and that of family members, and students' occupation with COVID-19. These decreases were visible especially in the first three days of our EMA period, and have also been observed in other studies, such as the COVID-19 social study that identified substantial drops within the first two days of assessment (https://www.marchnetwork.org/research). How can such decreases in our data be explained, given the rapid increases in infections and deaths in the Netherlands (Figure 1)? The most plausible interpretation is that the initial decrease represents a quick recovery towards baseline after a brief elevation, implying that we may have missed the peak a few days before. This is consistent with a 14-day EMA study in US students revealing short-lived increases in adverse mental health outcomes after the 2016 presidential election (Roche \& Jacobson, 2019). It is also in line with the COVID19related data published by the Understanding America Study, which monitors around 9,000 participants throughout the US; they recorded peaks of depression, anxiety, and stress between end of March and mid-April (https://covid19pulse.usc.edu/). Another study that examined US Google searches for mental health outcomes during the week of March 16 2020, coinciding with first week of our EMA data (Jacobson et al., 2020), identified considerable initial increases in mental health related searches, but a quick stabilization of searches in less than 4 days. A second interpretation is that the initial decrease could be artifactual, in the sense that early self-report items could be overreported. This has been described as initial elevation bias (Shrout et al., 2018). However, recent systematic investigations have raised doubts about initial elevation bias in the context of EMA. In a daily diary study of over 1,300 participants, Arslan et al. (2020) assessed constructs similar to those included in our study (e.g. stress, loneliness, mood), and concluded that findings are likely substantive in nature, rather than measurement artifacts.

Two findings stand out from the overall stable or decreasing mental health problems in our study. Participants reported at the exit measurement point on a 1-item screener that the pandemic had affected their mental health somewhat adversely; the specific item was: "The coronavirus (COVID-19) situation had an impact on my mental health (e.g. stress, anxiety, depressed mood)". The most plausible explanation for this divergent result, in our opinion, is students reported changes over a longer period of time than our study period, i.e. the pandemic had effected their mental health adversely before the start of our study. The result could also be a consequence of measurement: it was the only mental health item about retrospective change. A final explanation is that students answered this item about mental health problems other than stress, anxiety, or depression (although we provided these three as examples in the question), meaning that the item queried different content our main outcome measure, the DASS-21.

The second mental health finding that stands out from our results is the significant increase on the DASS-21 depression subscale from baseline to exit survey, when the anxiety subscale decreased, and the stress subscale remained stationary. We performed post-hoc analyses to investigate this result in more detail, by looking at which particular items are responsible for the increases in depression and decreases in anxiety (see supplementary materials for all individual item changes). We identified two items that drove the decrease for DASS-21 anxiety; both were related to panic or panic attacks (worried about situations one might panic in, and feeling close to panic). For the depression subscale, the two items that increased substantially over time were difficulty to work up initiative, and having nothing to look forward to. While we could not find similar results in the literature, these results do not appear counterintuitive to us: the pandemic initially increased panic-related thoughts, feelings, and behaviors, which quickly return to baseline in a fairly healthy sample of students. At the same time, closure of university and social distancing measures have a negative impact on motivation and future outlook. A related result to discuss is that the future outlook item on the DASS-21 depression subscale (nothing to look forward to) increased over time from pre to post, as just discussed, but we identified a significant decrease in the same item during our EMA, as shown in Table 1. In other words, the item increases over two repeated assessments two weeks apart, when asked how students felt in the last week, but decreases when monitoring it over 56 measurement points with the question how they felt in the last 3 hours. This is not necessarily contradictory, because these assessments cover different time frames: the baseline survey queried participants about the prior week, and EMA data collection started after that. This is consistent with the notion that we missed an early peak of mental health problems shortly before 
the beginning of our EMA study period: depression symptoms increased from week 1 to week 3 of our data collection in pre-post surveys, but decreased in EMA items from week 2 to 3 . Given the scarcity of measurement work on EMA, we do not know whether there might be other causes of this pattern in the data. It is possible that contemporaneous reporting differs from retrospective reporting of information in important ways, highlighting the importance of more focused measurement work in the domain of EMA research.

Regarding social contact, students reported decreasing levels of loneliness, both in EMA data and when comparing baseline and exit surveys. Additionally, students reported no changes in their amount of meaningful in-person social activities, and slight increases in their time staying at home (potentially spending more time with family, flat mates, and close friends). At the exit survey, students reported retrospectively that they had somewhat avoided social contact in larger groups during our study. Together, these findings imply that while overall in-person social contact remained fairly stable, the type of social contact may have changed to smaller -and potentially more meaningful-social interactions, which could explain the decreases in loneliness. This is consistent with the authors' own experiences: we were more in touch with our families during the outbreak of the pandemic in the Netherlands. During the SARS epidemic, adults in Hong Kong reported increases regarding 'feeling part of the community' compared to an assessment a year before the outbreak (Lau et al., 2008), which may be consistent with our findings of decreasing loneliness. While some additional measures were initiated by the Dutch government halfway through our EMA data collection period, such as limiting visitors to a maximum of 3 (see methods section for a more detailed description), the government had already announced some measures shortly before EMA data collection started, such as closing of universities. Students may therefore have decreased their social contacts in the days leading up to the beginning of our data collection, explaining why social contact may not have (further) during our data collection period.

Finally, the main results of the two network models were that mental health items clustered together and yielded some vicious cycles; that loneliness was positively related to mental health problems and concerns about COVID-19, which in turn predicted mental health outcomes; and that being outdoors was related to meaningful in-person social activities. There was some evidence for small peaks of state mental health problems on days 4 and 8, following university and government announcements. From a dynamical systems perspective, these peaks can be thought of as perturbations of the students' mental health systems caused by events in the external field (Borsboom, 2017; Robinaugh et al., 2019). This view offers the possibility that, especially in vulnerable students, timely interventions on elements of the dynamical system may have positive outcomes and prevent transitions into more severe problems. This is especially the case for elements that are part of vicious cycles, such as social contact, loneliness, or worry. Overall, future work should utilize time series data to investigate dynamical systems of mental health during stressors, and the potential benefits of prevention and intervention strategies targeting such systems using methods like control theory (Fried \& Robinaugh, 2020; Henry et al., 2020).

Before concluding this paper with limitations, we want to reiterate that many variables were assessed in the dataset that have not been analyzed for this report; that the all data, code, and measures are available online (https://osf.io/mvdpe/); and that we encourage others to use the data for their own work (see e.g. (Taquet et al., 2020)).

\section{Limitations and future directions}

Our study comes with a number of limitations. First, we focused our investigation on the group level, and only explored some inter-individual differences, via random effects multilevel regression models described in detail in the supplementary materials. One interesting result is the heterogeneity across participants in (univariate) auto-regressive effects for the majority of EMA variables. Idiographic follow-up work will be important to understand how time series of mental health data, and their relations, differ across people (Fisher et al., 2018; Fried \& Cramer, 2017; Hebbrecht et al., 2020). The second set of limitations pertains to measurement. To limit the burden of an already time-intensive study for students, and to minimize dropout, we shortened or adapted some scales, such as the loneliness scale, and were unable to assess many variables of interest in more detail, such as socioeconomic status or the nature of social contacts. Further, to keep surveys brief, we did not provide detailed definitions of the constructs we measured, such as meaningful inperson activities. This means that participants may interpret items differently. A study in 4,600 participants found that certain types of social interactions were considered to be especially 
meaningful (Litt et al., 2020), and that these results were consistent across several cultures. Meaningful interactions included those that enhanced people's lives, the lives of their interaction partners, or personal relationships; interactions with friends, family, or partners with community ties (such as neighbors); and interactions featuring shared activities, planned in advance, or memorialized via photographs or other media. Another limitation is that we created the EMA items for our study, given that there is no dedicated validation work in the context of EMA measurement as of yet. We urge the field to come together and initiate concerted efforts to develop valid and reliable scales suitable for investigate short-term dynamics of emotions and state mental health problems. Third, our study is limited in terms of measurement points, and we hope that future research on compliance rates in EMA data may elucidate ways to reliably gather data over longer assessment periods. Combining longer EMA periods with passive sensing and digital phenotype data collection via wearable devices may provide more fine-grained and objective data than we were able to collect (Jacobson et al., 2019). Finally, while the sample is a diverse sample in terms of nationalities, it is largely limited to European students, and is a convenience sample of psychology bachelor students living in the Netherlands. The Netherlands is a first world country, and has scored rank 1 in Europe several times in the last decade in terms of healthcare consumer satisfaction (Watson, 2012). It is an open question how well our results generalize to other populations, given the nature of the COVID-19 pandemic. Similarly, we focused on the immediate impact of the pandemic on student mental health. Other work has focused on longer-term consequences, such as a US-based study demonstrating increases in depression and anxiety symptoms in bachelor students in the winter semester 2020 compared to prior academic terms (Huckins et al., 2020).

\section{Open Practices Statement}

The study was not formally preregistered. We made all data, measures, and code available online: https://osf.io/mvdpe/

\section{Acknowledgements}

We thank all participants in the study, and all students involved in the data collection efforts for this project: Patrick Aigner, Anika Busch, Rayka Zavaro Dalva, Sophie Janken, Agnieszka Kusal, Lynn Mayer, Alice Micale, Giulia Murgia, Arthur de Souza Queiroz, Joe Saville, Luana Schehr, Emilie Sutter, Jonas Totland, Lea Velthuysen, Anna Wickenkamp, and Elias Zitterbarth. Special thanks to Leonie Cloos and Ricarda Proppert for their help in setting up the study. Thanks to Brenton Wierniek for the preprint template https://osf.io/hsv6a/.

\section{References}

Arslan, R. C., Reitz, A. K., Driebe, J. C., Gerlach, T. M., \& Penke, L. (2020). Routinely Randomize Potential Sources of Measurement Reactivity to Estimate and Adjust for Biases in Subjective Reports. Psychological Methods. https://doi.org/10.1037/met0000294

Auerbach, R. P., Alonso, J., Axinn, W. G., Cuijpers, P., Ebert, D. D., Green, J. G., Hwang, I., Kessler, R. C., Liu, H., Mortier, P., Nock, M. K., Pinder-Amaker, S., Sampson, N. A., Aguilar-Gaxiola, S., Al-Hamzawi, A., Andrade, L. H., Benjet, C., Caldas-De-Almeida, J. M., Demyttenaere, K., ... Bruffaerts, R. (2016). Mental disorders among college students in the World Health Organization World Mental Health Surveys. Psychological Medicine, 46(14), 2955-2970. https://doi.org/10.1017/S0033291716001665

Besser, A., \& Zeigler-hill, V. (2014). Positive Personality Features and Stress among First-year University Students : Implications for Psychological Distress, Functional Impairment , and. 13(1), 2444.

Bolger, N., Zuckerman, A., \& Kessler, R. C. (2000). Invisible support and adjustment to stress. Journal of Personality and Social Psychology, 79(6), 953-961. https://doi.org/10.1037/00223514.79.6.953

Borsboom, D. (2017). A network theory of mental disorders. World Psychiatry, 16, 5-13. https://doi.org/10.1002/wps.20375 
Cohen, S., \& Williamson, G. (1988). Perceived stress in a probability sample of the United States. In S. S. A. S. Oskamp (Ed.), The Social Psychology of Health (pp. 31-67). Sage.

Daly, M., Sutin, A., \& Robinson, E. (2020). Longitudinal changes in mental health and the COVID19 pandemic: Evidence from the UK Household Longitudinal Study. Psychological Medicine, 10-19. https://doi.org/10.1017/S0033291720004432

Denovan, A., \& Macaskill, A. (2016). Stress and Subjective Well-Being Among First Year UK. Journal of Happiness Studies. https://doi.org/10.1007/s10902-016-9736-y

Ebert, D. D., Auerbach, R., Cuijpers, P., Demyttenaere, K., Buntrock, C., Weisel, K. K., Green, J. G., Bruffaerts, R., Mortier, P., Kessler, R. C., Kiekens, G., \& Nock, M. K. (2018). Prediction of major depressive disorder onset in college students. Depression and Anxiety, 1-11. https://doi.org/10.1002/da.22867

Epskamp, S., Waldorp, L. J., Mõttus, R., \& Borsboom, D. (2018). The Gaussian Graphical Model in Cross-Sectional and Time-Series Data. Multivariate Behavioral Research, 53(4), 453-480. https://doi.org/10.1080/00273171.2018.1454823

Ettman, C. K., Abdalla, S. M., Cohen, G. H., Sampson, L., Vivier, P. M., \& Galea, S. (2020). Prevalence of Depression Symptoms in US Adults Before and During the COVID-19 Pandemic. JAMA Network Open, 3(9), e2019686. https://doi.org/10.1001/jamanetworkopen.2020.19686

Fisher, A. J., Medaglia, J. D., \& Jeronimus, B. F. (2018). Lack of group-to-individual generalizability is a threat to human subjects research. Proceedings of the National Academy of Sciences, 201711978. https://doi.org/10.1073/pnas.1711978115

Frank, E., Nallamothu, B. K., Zhao, Z., \& Sen, S. (2019). Political events and mood among young physicians: A prospective cohort study. The BMJ, 367. https://doi.org/10.1136/bmj.16322

Fried, E. I., \& Cramer, A. O. J. (2017). Moving forward: challenges and directions for psychopathological network theory and methodology. Perspectives on Psychological Science, 12(6), 999-1020. https://doi.org/10.1177/1745691617705892

Fried, E. I., Nesse, R. M., Zivin, K., Guille, C., \& Sen, S. (2014). Depression is more than the sum score of its parts: individual DSM symptoms have different risk factors. Psychological Medicine, 44, 20672076. https://doi.org/10.1017/S0033291713002900

Fried, E. I., \& Robinaugh, D. J. (2020). Systems all the way down: embracing complexity in mental health research. BMC Medicine, 18(205), 1-4. https://doi.org/10.1186/s12916-020-01668-w

Gaspersz, R., Frings-dresen, M. H. W., \& Sluiter, J. K. (2012). Prevalence of common mental disorders among Dutch medical students and related use and need of mental health care: a crosssectional study. International Journal of Adolescent Medicine and Health, 24(2), 169-172. https://doi.org/10.1515/IJAMH.2012.025

Granger, C. W. J. (1969). Investigating Causal Relations by Econometric Models and Cross-spectral Methods. Econometrica, 37(3), 424-438. https://doi.org/10.2307/1912791

Guthrie, E., Black, D., Creed, F., \& Shaw, C. (1998). Stress and Burnout in Medical Students: Journal of the Royal Society of Mendicine, 91, 237-243.

Hawryluck, L., Gold, W. L., Robinson, S., Pogorski, S., Galea, S., \& Styra, R. (2004). SARS control and psychological effects of quarantine, Toronto, Canada. Emerging Infectious Diseases, 10(7), 12061212. https://doi.org/10.3201/eid1007.030703

Hebbrecht, K., Stuivenga, M., Birkenhäger, T., Morrens, M., Fried, E. I., Sabbe, B., \& Giltay, E. J. (2020). Understanding personalized dynamics to inform precision medicine : a dynamic time warp analysis of 255 depressed inpatients. BMC Medicine, 1-15.

Heckman, S., Lim, H., \& Montalto, C. (2014). Factors Related to Financial Stress among College Students. 5(1).

Henry, T. R., Robinaugh, D. J., \& Fried, E. (2020). On the control of psychological networks. 1-30.

Huckins, J. F., da Silva, A. W., Wang, W., Hedlund, E., Rogers, C., Nepal, S. K., Wu, J., Obuchi, M., Murphy, E. I., Meyer, M. L., Wagner, D. D., Holtzheimer, P. E., \& Campbell, A. T. (2020). Mental health and behavior of college students during the early phases of the COVID-19 pandemic: Longitudinal smartphone and ecological momentary assessment study. Journal of Medical Internet Research, 22(6). https://doi.org/10.2196/20185

Jacobson, N., Lekkas, D., Price, G., Veinz, M., Song, M., O’Malley, A., \& Barr, P. (2020). Flattening the Mental Health Curve: COVID-19 Stay-at-Home Orders Result in Alterations in Mental Health Search Behavior in the United States.

Jacobson, N., Weingarden, H., \& Wilhelm, S. (2019). Digital biomarkers of mood disorders and symptom change. Npj Digital Medicine, 2(1), 88-90. https://doi.org/10.1038/s41746-019-0078-0 
Kessler, R. C., Chiu, W. T., Demler, O., Merikangas, K. R., \& Walters, E. E. (2005). Prevalence, severity, and comorbidity of 12-month DSM-IV disorders in the National Comorbidity Survey Replication. Archives of General Psychiatry, 62(6), 617-627. https://doi.org/10.1001/archpsyc.62.6.617

Kiekens, G., Hasking, P., Claes, L., Boyes, M., Mortier, P., Auerbach, R. P., Cuijpers, P., Demyttenaere, K., Green, J. G., Kessler, R. C., Myin-Germeys, I., Nock, M. K., \& Bruffaerts, R. (2019). Predicting the incidence of non-suicidal self-injury in college students. European Psychiatry, 59, 44-51. https://doi.org/10.1016/j.eurpsy.2019.04.002

Kwong, A. S. F., Northstone, K., Pearson, R. M., Smith, D., Lawlor, D. A., \& Timpson, N. J. (2020). Longitudinal evidence for persistent anxiety in young adults through COVID-19 restrictions [ version 1; peer review : 2 approved with reservations ]. 1-10.

Lau, A. L. D., Chi, I., Cummins, R. A., Lee, T. M. C., Chou, K. L., \& Chung, L. W. M. (2008). The SARS (Severe Acute Respiratory Syndrome) pandemic in Hong Kong: Effects on the subjective wellbeing of elderly and younger people. Aging and Mental Health, 12(6), 746-760. https://doi.org/10.1080/13607860802380607

Litt, E., Zhao, S., Kraut, R., \& Burke, M. (2020). What Are Meaningful Social Interactions in Today's Media Landscape? A Cross-Cultural Survey. Social Media and Society, 6(3). https://doi.org/10.1177/2056305120942888

Lovibond, P., \& Lovibond, S. (1995). The structure of negative emotional states: comparison of the depression anxiety stress scales (DASS) with the back depression and anxiety inventories. Behavior Research and Therapy, 33(3), 335-343.

Luijten, M. A. J., van Muilekom, M. M., Teela, L., van Oers, H. A., Terwee, C. B., Zijlmans, J., Klaufus, L., Popma, A., Oostrom, K. J., Polderman, T. J. C., \& Haverman, L. (2020). The impact of lockdown during the COVID-19 pandemic on mental and social health of children and adolescents. MedRxiv, 2020.11.02.20224667.

http://medrxiv.org/content/early/2020/11/04/2020.11.02.20224667.abstract

Mackenzie, S., Wiegel, J. R., Mundt, M., Brown, D., Saewyc, E., Heiligenstein, E., Harahan, B., \& Fleming, M. (2011). Depression and Suicide Ideation Among Students Accessing Campus Health Care. 81(1), 101-107. https://doi.org/10.1111/j.1939-0025.2010.01077.x

McGinty, E. E., Presskreischer, R., Anderson, K. E., Han, H., \& Barry, C. L. (2020). Psychological Distress and COVID-19-Related Stressors Reported in a Longitudinal Cohort of US Adults in April and July 2020. JAMA - Journal of the American Medical Association, July. https://doi.org/10.1001/jama.2020.21231

Misra, R., \& McKean, M. (2000). College students' academic stress and its relation to their anxiety, time management, and leisure satisfaction. American Journal of Health Studies.

Montgomery, M., \& Cote, J. (2003). College as a transition to adulthood. In G. R. Adams \& M. D. Berzonsky (Eds.), Blackwell handbooks of developmental psychology. Blackwell handbook of adolescence (pp. 149-172). Blackwell Publishing.

Mortier, P., Auerbach, R. P., Alonso, J., Bantjes, J., Benjet, C., Cuijpers, P., Ebert, D. D., Green, J. G., Hasking, P., Nock, M. K., Neill, S. O., Pinder-amaker, S., Sampson, N. A., Vilagut, G., Zaslavsky, A. M., Bruffaerts, R., Kessler, R. C., \& Collaborators, W. H. O. W. (2018). Suicidal Thoughts and Behaviors Among First-Year College Students: Results From the WMH-ICS Project. Journal of the American Academy of Child \& Adolescent Psychiatry. https://doi.org/10.1016/j.jaac.2018.01.018

Mortier, P., Demyttenaere, K., Auerbach, R. P., Cuijpers, P., Green, J. G., Kiekens, G., Kessler, R. C., Nock, M. K., Zaslavsky, A. M., \& Bruffaerts, R. (2018). First onset of suicidal thoughts and behaviours in college. Journal of Affective Disorders, 207, 291-299. https://doi.org/10.1016/j.jad.2016.09.033.FIRST

Nelson, B. W., Pettitt, A., Flannery, J., \& Allen, N. B. (2020). Psychological and Epidemiological Predictors of COVID-19 Concern and Health-Related Behaviors.

Ribeiro, I. J. S., Pereira, R., Freire, I. V, Oliveira, B. G. De, Casotti, C. A., \& Boery, E. (2017). Stress and quality of life among university students: a systematic literature review. Health Professions Education. https://doi.org/10.1016/j.hpe.2017.03.002

Robinaugh, D. J., Hoekstra, R. H. A., Toner, E. R., \& Borsboom, D. (2019). The network approach to psychopathology: a review of the literature 2008-2018 and an agenda for future research. Psychological Medicine. https://doi.org/10.1080/01559982.2019.1584953 
Roche, M. J., \& Jacobson, N. (2019). Elections Have Consequences for Student Mental Health: An Accidental Daily Diary Study. Psychological Reports, 122(2), 451-464. https://doi.org/10.1177/0033294118767365

Russell, D., Peplau, L. A., \& Cutrona, C. E. (1980). The revised UCLA Loneliness Scale: Concurrent and discriminant validity evidence. Journal of Personality and Social Psychology, 39(3), 472-480. https://doi.org/10.1037/0022-3514.39.3.472

Schwarzer, R., \& Jerusalem, M. (1995). Generalized Self-Efficacy scale. In J. Weinman, S. Wright, \& M. Johnston (Eds.), Measures in health psychology: A user's portfolio. Causal and control beliefs (pp. 35-37). NFER-NELSON.

Shrout, P. E., Stadler, G., Lane, S. P., Joy McClure, M., Jackson, G. L., Clavél, F. D., Iida, M., Gleason, M. E. J., Xu, J. H., \& Bolger, N. (2018). Initial elevation bias in subjective reports. Proceedings of the National Academy of Sciences of the United States of America, 115(1), E15-E23. https://doi.org/10.1073/pnas.1712277115

Spitzer, R. L., Kroenke, K., Williams, J. B. W., \& Löwe, B. (2006). A brief measure for assessing generalized anxiety disorder: The GAD-7. Archives of Internal Medicine, 166(10), 1092-1097. https://doi.org/10.1001/archinte.166.10.1092

Stallman, H. M. (2010). Psychological distress in university students : A comparison with general $\begin{array}{llll}\text { population data. Australian 249-257. } & \text { Psychologist, 45), }\end{array}$ https://doi.org/10.1080/00050067.2010.482109

Taquet, M., Quoidbach, J., Fried, E., \& Goodwin, G. M. (2020). Mood Homeostasis Before and During the Coronavirus Disease 2019 (COVID-19) Lockdown Among Students in the Netherlands. JAMA Psychiatry, 2019, 2019-2021. https://doi.org/10.1001/jamapsychiatry.2020.2389

The Dutch Government. (2020). The approach to tackling coronavirus in the Netherlands. Government.Nl. https://www.government.nl/topics/coronavirus-covid-19/tackling-newcoronavirus-in-the-netherlands

Tomoda, A., Mori, K., \& Kimura, M. (2000). One-year prevalence and incidence of depression among first-year university students in Japan: A preliminary study. Psychiatry and Clinical Neurosciences (2000), 54, 583-588.

Tran, A., Tran, L., Geghre, N., Darmon, D., Rampal, M., Rebouillat-savy, K., Brandone, D., Gozzo, J., \& Avillach, P. (2017). Health assessment of French university students and risk factors associated with mental health disorders. 1-18. https://doi.org/10.5061/dryad.54qt7

Twenge, J. M., \& Joiner, T. E. (2020). U.S. Census Bureau-assessed prevalence of anxiety and depressive symptoms in 2019 and during the 2020 COVID-19 pandemic. Depression and Anxiety, 37(10), 954-956. https://doi.org/10.1002/da.23077

Watson, R. (2012). Netherlands tops European healthcare league, with UK coming in at 12th. BMJ (Clinical Research Ed.), 344(May), 1-2. https://doi.org/10.1136/bmj.e3430

Wilks, S. E., Spivey, C. A., Wilks, S. E., \& Spivey, C. A. (2009). The Resilience in Undergraduate Social Work Students: Social Support and Adjustment to Academic Stress. Social Work Education: The International Journal, 37-41. https://doi.org/10.1080/02615470902912243

Williams, C. J., Dziurawiec, S., \& Heritage, B. (2018). More Pain Than Gain: Effort - Reward Imbalance, Burnout, and Withdrawal Intentions Within a University Student Population. Journal of Educational Psychology, 110(3), 378-394. 